\title{
Consequentes de Motivação do Serviço Público: Proposição de um Framework de Análise em Organizações Públicas Brasileiras
}

Menezes de Oliveira, Jefferson; Barros Estivalete, Vania de Fátima

Consequentes de Motivação do Serviço Público: Proposição de um Framework de Análise em Organizações

Públicas Brasileiras

Administração Pública e Gestão Social, vol. 11, núm. 4, 2019

Universidade Federal de Viçosa, Brasil

Disponível em: http://www.redalyc.org/articulo.oa?id=351560525004

Esta obra está bajo una Licencia Creative Commons Atribución-NoComercial-SinDerivar 3.0 Internacional. 


\title{
Consequentes de Motivação do Serviço Público: Proposição de um Framework de Análise em Organizações Públicas Brasileiras
}

\begin{abstract}
Public Service Motivation Consequences: Proposition of an Analysis Framework in Brazilian Public Organizations Consecuencias de Motivación del Servicio Público: Proposición de un Framework de Análisis en Organizaciones Públicas Brasileñas
\end{abstract}

Jefferson Menezes de Oliveira

Universidade Federal de Santa Maria, Brasil

Redalyc: http://www.redalyc.org/articulo.oa?

jefferson@ufsm.br

Vania de Fátima Barros Estivalete

Universidade Federal do Rio Grande do Sul, Brasil

vaniaestivalete@ufsm.br

Recepção: 17 Janeiro 2019

Aprovação: 13 Agosto 2019

Publicado: 01 Outubro 2019

\section{Resumo:}

O presente trabalho tem por objetivo propor um framework de análise para a Motivação do Serviço Público (MSP) em organizações do setor público brasileiro, mais especificamente explorando seus reflexos sobre o Ajuste Indivíduo-Organização (AIO) e o Comportamento de Cidadania Organizacional (CCO). O modelo oferecido apresenta proposições elaboradas a partir da teoria e, uma vez validado empiricamente, possibilitará investigar as influências da MSP sobre a percepção de congruência de valores entre indivíduo e organização e, também, sobre a propensão à manifestação de comportamentos discricionários que podem ser positivos ao ambiente organizacional.

Palavras-Chave: Motivação do Serviço Público, Framework de Análise, Consequentes.

\begin{abstract}
:
This paper aims to propose an analytical framework for Public Service Motivation (PSM) in organizations of the Brazilian public sector, specifically exploring its impacts on the Person-Organization Fit (P-O Fit) and Organizational Citizenship Behavior (OCB). The model offered presents propositions elaborated from the theory and once empirically validated, will allow to investigate the influences of PSM on the perception of congruence between individual and organization values and also on the propensity to the manifestation of discretionary behaviors that can be positive to the organizational environment.
\end{abstract}

KEYWORDS: Public Service Motivation, Analysis Framework, Consequences.

\section{ReSUMEN:}

El presente trabajo tiene como objetivo proponer un framework de análisis para la Motivación del Servicio Público (MSP) en organizaciones del sector público brasileño, explorando específicamente sus reflejos sobre el Ajuste Individuo-Organización (AIO) y el Comportamiento de Ciudadanía Organizacional (CCO). El modelo ofrecido presenta proposiciones elaboradas a partir de la teoría y, una vez validado empíricamente, permitirá investigar las influencias de la MSP sobre la percepción de congruencia de valores entre individuo y organización y, también, sobre la propensión a la manifestación de comportamientos discrecionales que pueden ser positivos al ambiente organizacional.

Palabras Clave: Motivación para el Servicio Público, Framework de Análisis, Consecuencias.

\section{INTRODUÇÃO}

Em organizações públicas, uma das importantes questões de pesquisa e desafios práticos na área de gestão de pessoas e comportamento organizacional é compreender o processo de motivação de seus trabalhadores 
(Park \& Rainey, 2008). Nesse sentido, o conceito de Motivação do Serviço Público (MSP), ou Public Service Motivation (PSM), surge como uma lente pela qual gestores e pesquisadores poderão ampliar tal compreensão.

O conceito de MSP logrou difusão a partir da publicação do artigo “The Motivational Bases of Public Service”, de autoria dos pesquisadores Perry e Wise (1990). Inicialmente, a MSP foi concedida como uma predisposição individual para responder a motivações fundamentadas, principalmente ou exclusivamente, em instituições e organizações públicas (Perry \& Wise, 1990). Após refinamento conceitual, Kim et al. (2013) inferem que a MSP se refere a um tipo de motivação pró-social que impulsiona os indivíduos a trabalharem pelo bem comum, comprometidos com os valores públicos, com empatia e solidariedade aos demais indivíduos e sujeitos a sacrifícios pessoais em prol da sociedade.

Internacionalmente, são crescentes os estudos que abordam os consequentes de MSP nas organizações, tais como sua influência na escolha pelo emprego público (Vandenabeele, 2008; Clerkin \& Coggburn, 2012; Carpenter, Doverspike \& Miguel, 2012), nas percepções de ajuste entre indivíduo e organização (Bright, 2008; Kim, 2012; Bright, 2013), nas atitudes gerais que são benéficas para o desempenho organizacional, tais como o comprometimento organizacional e a satisfação no trabalho (Taylor, 2008; Kim, 2012; Ritz, 2009) e em comportamentos específicos, tais como o comportamento de cidadania organizacional - CCO (Cun, 2012; Ferdousipour, 2016; Gould-Williams, Mostafa, \& Bottomley, 2015; Kim, 2006; Koumenta, 2015).

O presente estudo busca explorar os reflexos da MSP sobre percepções e comportamentos considerados profícuos às organizações públicas por meio da proposição de um modelo inovador ao contexto brasileiro. Sendo assim, busca-se propor um framework de análise da motivação do serviço público e suas influências sobre as percepções de ajuste entre indivíduo e organização e o comportamento de cidadania organizacional em organizações públicas brasileiras.

O Ajuste Indivíduo-Organização (AIO), opção de tradução para a expressão Person-Organization Fit (P-O Fit), ocorre quando os funcionários acreditam que seus valores são congruentes com os valores da organização (Cable \& Derue, 2002). Em estudos internacionais, além de ser considerado um consequente direto da MSP, o AIO é sinalizado como um possível elemento mediador na interação entre a MSP e outros resultados, tais como a atração para organizações do setor público (Carpenter et al., 2012), a satisfação no trabalho (Bright, 2008; Kim, 2012; Steijn, 2008; Wright \& Pandey, 2008), o comprometimento organizacional (Kim, 2012), a permanência no trabalho atual (Steijn, 2008; Bright, 2008) e o CCO (GouldWilliams et al., 2015).

Em relação ao CCO, ressalta-se que o contexto de trabalho, além de abarcar tarefas relacionadas ao desempenho de funções relativas ao núcleo técnico da organização, compreende facetas variáveis de comportamento discricionário que podem propiciar um cenário organizacional positivo (Becton, Carr, Mossholder, \& Walker, 2017). Enquanto comportamento discricionário que não é reconhecido pelo sistema formal de recompensas, o CCO pode contribuir para a eficácia organizacional (Organ, 1988), sobretudo por meio de suas contribuições para a manutenção e aprimoramento do contexto social e psicológico que dá suporte ao desempenho das tarefas (Organ, 1997). Nesse contexto, pressupõe-se que indivíduos voltados ao interesse público irão visualizar seu trabalho de forma mais ampla, despendendo esforços que superem suas atividades rotineiras e formais. Sendo assim, é provável que indivíduos com MSP manifestem o CCO com mais frequência (Koumenta, 2015).

De acordo com o estudo de Gould-Williams et al. (2015), realizado no contexto egípcio, os efeitos positivos da MSP sobre o CCO foram, além de diretos, mediados pela percepção de congruência entre os valores dos trabalhadores e os da organização. Perante o exposto, postula-se que a MSP pode influenciar diretamente o $\mathrm{AIO}$ e o $\mathrm{CCO}$ nas organizações públicas brasileiras. Complementarmente, presume-se a existência de um efeito indireto da MSP sobre o CCO por intermédio do AIO.

O presente trabalho, portanto, tem por objetivo propor um framework de análise da MSP para o setor público brasileiro, mais especificamente explorando seus reflexos sobre o AIO e o CCO. Compreende-se 
que o referido setor possui componentes próprios, os quais tornam desafiadores os trabalhos relacionados ao comportamento humano. De acordo com Bergue (2010), são condicionantes, dentre outras, as normativas das relações entre administração e agente público, a natureza dos bens e serviços oferecidos, a dinâmica das atividades internas ao ambiente de trabalho e as relações pessoais. Por isso, os administradores públicos devem ampliar suas capacidades de compreender os mecanismos motivacionais e de comportamento dos indivíduos em ambientes organizacionais com pujantes traços burocráticos e patrimonialistas.

Conforme Bright (2008), uma vez identificada a associação entre a MSP e resultados positivos para as organizações públicas, o construto pode ser concebido como um guia para socializar e treinar funcionários. Além disso, este estudo é justificado pelo oferecimento de uma agenda de pesquisa para as áreas de administração pública e comportamento organizacional no contexto brasileiro. O trabalho segue estruturado com as seções de fundamentação teórica, apresentação do framework proposto e considerações finais.

\section{FUNDAMENTAÇÃO TEÓRICA}

Esta seção tem por intuito apresentar concepções teóricas sobre o construto Motivação do Serviço Público (MSP), bem como seus principais consequentes identificados na literatura internacional, com ênfase no Ajuste Indivíduo-Organização (AIO) e no Comportamento de Cidadania Organizacional (CCO). As concepções trazidas pela literatura constituíram os alicerces para a proposição do framework de análise apresentado na seção subsequente.

\subsection{Motivação do Serviço Público (MSP)}

Até a década de 1980, a noção de motivação específica associada ao serviço público não havia logrado clareza conceitual. Era tratada de forma ampla e multifacetada e até mesmo confundida com outros construtos (Buchanan, 1975; Rainey, 1982). De forma a preencher tal lacuna conceitual, uma definição mais robusta e consistente de MSP foi desenvolvida somente na década de 1990, quando Perry e Wise (1990) a definiram como uma predisposição individual para responder a motivações fundamentadas, principalmente ou exclusivamente, em instituições e organizações públicas.

A partir das concepções de Knoke e Wright-Isak (1982), Perry e Wise (1990) identificaram três bases teóricas ou "motivos" da MSP: Racional, normativo e afetivo. Em primeiro lugar, os motivos racionais baseiam-se na maximização da utilidade individual e são manifestados quando os indivíduos querem participar do processo político, estão comprometidos e identificados com um programa público e servem como defensores de um interesse especial ou privado. Em segundo lugar, os motivos normativos se baseiam no desejo de perseguir o bem comum e, além disso, o interesse público. Esses motivos incluem patriotismo, dever e lealdade ao governo. Em terceiro lugar, os motivos afetivos são fundamentados na emoção humana e são caracterizados por um desejo e disposição para ajudar os outros (Perry \& Wise, 1990; Knoke \& WrightIsak, 1982).

Em trabalho posterior, a partir de tais "motivos" e da noção de auto sacrifício, Perry (1996) identificou e operacionalizou um conjunto de vinte e quatro (24) itens distribuídos em quatro dimensões distintas para mensurar a MSP: (i) Atração para a elaboração de políticas públicas, (ii) comprometimento com o interesse público/dever cívico, (iii) compaixão e (iv) auto sacrifício (Perry \& Wise, 1990; Camilleri, 2007; Wright \& Pandey, 2008).

Os estudos pioneiros de Perry e Wise (1990) e Perry (1996) fizeram com que surgisse uma nova agenda de pesquisa internacional em administração pública, tornando a MSP um construto alvo de constante refinamento conceitual. Brewer e Selden (1998), por exemplo, definiram a MSP como a força motivacional que induz os indivíduos a realizarem um serviço público significativo, sendo predominante entre a força 
de trabalho do setor público. Scott e Pandey (2005) apontaram que o conceito de MSP denota a ideia de comprometimento com o serviço público, a busca do interesse público e o desejo de realizar um trabalho oportuno para a sociedade. Kim (2006), por sua vez, complementou que a MSP é caracterizada por uma prevalência de recompensas intrínsecas sobre recompensas extrínsecas.

Kim e Vandenabeele (2010), em refinamento teórico posterior, inferem que a MSP é permeada pela noção de auto sacrifício e está associada aos motivos instrumental, baseado em valores e de identificação. Esses autores, portanto, apresentaram uma revisão das bases teóricas apontadas por Perry e Wise (1990), excluindo o interesse privado como componente de MSP.

Os motivos instrumentais são baseados no altruísmo e incluem trabalhar no setor público, participar do processo político e atividades comunitárias, participar de atividades de desenvolvimento social e defender políticas e programas públicos especiais, a fim de realizar um serviço público significativo para a sociedade. Motivos baseados em valores referem-se aos valores públicos finalísticos que os indivíduos desejam alcançar através de seus comportamentos e ações. Trata-se de uma internalização dos valores públicos, ou seja, os indivíduos podem considerar os valores públicos como seus próprios valores. Os valores públicos podem incluir o interesse público, a responsabilidade social, a democracia, a equidade social, a justiça social, a neutralidade e a responsabilidade. Os motivos de identificação dizem respeito a pessoas, grupos ou objetos que os indivíduos desejam servir. Esse processo de identificação traz a disposição para fazer o bem e até mesmo se sacrificar pelos elementos identificados. Esta base, portanto, enfatiza o comprometimento afetivo de um indivíduo ou preocupação com as necessidades de outros indivíduos ou grupos específicos (Kim \& Vandenabeele, 2010).

A partir das bases teóricas revisadas por Kim e Vandenabeele (2010), Kim et al. (2013) realizaram uma pesquisa em 12 países - Austrália, Bélgica, China, Dinamarca, França, Itália, Coreia do Sul, Lituânia, Holanda, Suíça, Reino Unido e Estados Unidos - com a finalidade de validar um instrumento internacional para mensurar a MSP. Com base no refinamento e esclarecimento de quatro dimensóes operacionais da MSP, foram gerados itens para mensurar a atração para o serviço público (ASP), o comprometimento com os valores públicos (CVP), a compaixão (COM) e o auto sacrifício (AS). O Quadro 01 apresenta as definições dessas dimensões. 
Quadro 01. Dimensões da escala internacional de MSP

\begin{tabular}{|l|l|}
\hline Dimensão & Descrição \\
\hline $\begin{array}{l}\text { Atração para o } \\
\text { Serviço Público } \\
\text { ASP) }\end{array}$ & $\begin{array}{l}\text { Dimensão que analisa a disposição de servir } \\
\text { ao publico de forma significativa, de } \\
\text { trabalhar pelo bem comum e de participar } \\
\text { do processo de desenvolvimento e } \\
\text { implementação de politicas públicas. }\end{array}$ \\
\hline $\begin{array}{l}\text { Comprometimento } \\
\text { com os Valores } \\
\text { Públicos (CVP) }\end{array}$ & $\begin{array}{l}\text { Dimensão que analisa a disposição pessoal } \\
\text { para buscar o interesse público e os valores } \\
\text { públicos. Envolve julgar importante a } \\
\text { igualdade de oportunidades e a prestação } \\
\text { contínua de serviços públicos, agir } \\
\text { eticamente e preocupar-se com as gerações } \\
\text { futuras. }\end{array}$ \\
\hline Compaixão (CoM) & $\begin{array}{l}\text { Dimensão que analisa a ligação afetiva com } \\
\text { outros membros de uma categoria social ou } \\
\text { de um sistema politico. Envolve ter empatia } \\
\text { e solidariedade com os menos favorecidos, } \\
\text { ser pautado pela justiça e considerar o } \\
\text { bem-estar dos outros muito importante. }\end{array}$ \\
\hline $\begin{array}{l}\text { Auto Sacrifício } \\
\text { (AS) }\end{array}$ & $\begin{array}{l}\text { Considerada uma dimensão que dá suporte } \\
\text { às demais, envolve a realização de } \\
\text { sacrifícios pessoais em prol da sociedade. } \\
\text { Essa dimensão é bastante semelhante à } \\
\text { existente na escala de Perry (1996). }\end{array}$ \\
\hline
\end{tabular}

Fonte: elaborado a partir de Kim et al. (2013).

Salienta-se que os países pesquisados por Kim et al. (2013) não diferiram quanto ao número ou à composição das quatro dimensões representadas no instrumento de mensuração da MSP, o que lhe conferiu uma invariância configural. No entanto, a força da relação entre cada dimensão e seus itens não foi a mesma em todos os 12 países, o que não conferiu invariância métrica ao instrumento. Por isso, Kim et al. (2013) apontam que o significado das dimensóes da MSP poderá diferir entre culturas e idiomas, sendo esse um ponto a ser considerado pelos pesquisadores que desejam verificar a validade da escala em novos contextos.

Kim (2017), ao ponderar que o estudo anterior (Kim et al., 2013) não alcançou a invariância métrica para a escala de MSP entre todas as culturas e línguas analisadas, desenvolveu um novo arranjo de itens para mensurar a MSP no contexto coreano, tendo em vista a obtenção das mesmas propriedades psicométricas entre diferentes grupos do país. Nessa revisão da escala internacional, Kim (2017) logrou confiabilidade, validade convergente e validade discriminante para um modelo de 29 itens distribuídos em quatro dimensões de análise para o construto (Atração para o Serviço Público - ASP, Comprometimento com os Valores Públicos - CVP, Compaixão - COM e Auto Sacrifício - AS).

Portanto, tendo em vista as recentes concepções teóricas, a MSP pode ser concebida como uma força que impulsiona os indivíduos a trabalharem pelo bem comum, comprometidos com os valores públicos, com empatia e solidariedade aos demais indivíduos e sujeitos a sacrifícios pessoais em prol da coletividade (Kim et al., 2013; Kim, 2017). Destaca-se que esse conceito possui relação complementar com a noção de vocação, sobretudo, por associar-se a um "chamado" atribuído ao indivíduo que deseja realizar um trabalho socialmente útil (Thompson \& Christensen, 2018).

Por fim, destaca-se que a pesquisa sobre MSP pode ser classificada em seis temas principais na atualidade: (i) A construção e sua mensuração, (ii) a incidência, (iii) os antecedentes, (iv) os resultados, (v) os sistemas organizacionais e (vi) a interação com outros tipos de motivação (Kim \& Vandenabeele, 2010). Considerando que o presente estudo tem por objetivo explorar reflexos da MSP no contexto do setor público brasileiro, a próxima subseção apresenta os principais consequentes identificados na literatura internacional. 


\subsection{Consequentes de MSP}

Em relação aos efeitos da MSP, identifica-se que o construto é considerado um antecedente de diversos fatores individuais e organizacionais. Preliminarmente, Perry e Wise (1990) levantaram a hipótese de que indivíduos com altos níveis de MSP teriam níveis significativamente maiores de satisfação no trabalho, desempenho e comprometimento em organizações públicas quando comparados com indivíduos com níveis mais baixos de MSP.

Considerando a literatura internacional, verifica-se que a MSP é amplamente associada à satisfação no trabalho (Naff \& Crum, 1999; Vandenabeele, 2007; Steijn, 2008; Taylor, 2008; Bright, 2008; Wright \& Pandey, 2008; Bangcheng, 2009; Georgellis \& Tabvuma, 2010; Kim, 2011; Kim, 2012; Cun, 2012; Taylor, 2014; Liu, Tang, \& Yang, 2015; Teo, Pick, Xerri, \& Newton, 2016; Liu \& Perry, 2016; Simone, Cicotto, Pinna, \& Giustiniano, 2016).

Além disso, verifica-se a constante associação da MSP aos consequentes: Comprometimento organizacional (Crewson, 1997; Taylor, 2008; Ritz, 2009, Kim, 2012; Caillier, 2015b; Riba \& Ballart, 2016; Caillier, 2016), comportamento de cidadania organizacional (Kim, 2006; Cun, 2012; Gould-Williams et al., 2015; Koumenta, 2015; Ferdousipour, 2016), comportamento extra papel (Caillier, 2015; Caillier, 2016), comportamento pró-social (Esteve, Urbig, Van Witteloostuijn, \& Boyne, 2016; Piatak, 2016), comportamento de cidadania interpessoal (Pandey, Wright, \& Moynihan, 2008), comportamento de cidadania comunitária (Liu, Zhang, Du, \& Hu, 2015; Liu \& Perry, 2016), comportamento ético/intenção de denunciar (Brewer \& Selden, 1998; Wright, Hassan, \& Park, 2016), identificação organizacional (Liu \& Perry, 2016), compartilhamento do conhecimento (Chen \& Hsieh, 2015; Tuan, 2016), engajamento no trabalho (Simone et al., 2016), propensão ao voluntariado (Houston, 2005; Clerkin, Paynter, \& Taylor, 2009; Piatak, 2016), escolha por trabalhar no setor público (Vandenabeele, 2008; Steijn, 2008; Clerkin \& Coggburn, 2012; Carpenter et al., 2012; Pedersen, 2013), intenção de permanecer na organização (Naff \& Crum, 1999; Bright, 2008, Steijn, 2008), ajuste indivíduo-organização (Bright, 2008; Kim, 2012; Bright, 2013), valência da missão institucional (Wright, Moynihan, \& Pandey, 2012; Caillier, 2015), aceitação de mudanças organizacionais (Wright, Christensen, \& Isett, 2013) e desempenho no trabalho (Bellé, 2013; Caillier, 2014; Cheng, 2015; Schwarz, Newman, Cooper, \& Eva, 2016; Van Loon, 2016).

O estudo meta-analítico de Harari, Herst, Parola e Carmona (2017) apontou que os indivíduos que tendem a valorizar o bem comum, em detrimento das necessidades individuais, criam um ajuste mais forte entre os objetivos pessoais e os objetivos organizacionais. Esse foco maior no coletivo em nível social não apenas cria um alinhamento entre os valores da organização e as necessidades individuais (Vandenabeele, 2007), mas também fortalece a satisfação no trabalho, pois os indivíduos são capazes de satisfazer suas necessidades pró-sociais por meio do trabalho (Bright, 2008).

Sendo assim, à medida que as organizações do setor público são encarregadas de promover o bem-estar geral da sociedade, prevê-se que a força de trabalho desse setor incluirá indivíduos com valores semelhantes, buscando oportunidades para satisfazer seus motivos altruístas para servir ao público. Sendo assim, é possível pressupor que indivíduos motivados para o serviço público estarão melhor ajustados em organizações públicas pela congruência de valores (Wright, 2007; Gould-Williams et al., 2015).

Nesse sentido, enquanto possível consequente de MSP, torna-se conexo o conceito de Ajuste IndivíduoOrganização (AIO), opção de tradução para a expressão Person-Organization Fit (P-O Fit). Esse tipo de ajuste ocorre "quando os funcionários acreditam que seus valores correspondem aos valores da organização e aos valores de outros funcionários da organização" (Cable \& Derue, 2002, p. 876), compondo uma das dimensões do ajuste entre indivíduo e ambiente de trabalho, ou Person-Environment Fit (P-E Fit), conceito oriundo da psicologia interacional.

Para avaliar o AIO existem formas diretas e indiretas. A primeira mensura o ajuste percebido, por correspondência ou semelhança, entre a pessoa e a organização. Nesse sentido, os entrevistados são 
questionados sobre o ajuste entre si e sua organização de forma direta (Kristof, 1996; Edwards et al., 2006; Cable \& Derue, 2002; Cable \& Judge, 1996; Saks \& Ashforth, 1997). As formas indiretas de avaliar o AIO, por sua vez, envolvem uma comparação entre características individuais e organizacionais separadamente (Kristof, 1996) e podem ser consideradas subjetivas ou objetivas.

Destaca-se a medida direta concebida por Cable e DeRue (2002), a qual considera três dimensões de ajuste. A primeira dimensão é centrada no AIO, enquanto uma congruência de valores, referindo-se ao aspecto suplementar ou ajuste por similaridade apoiado em estudos anteriores (Cable \& Judge, 1996; Chatman, 1989; Lauver \& Kristof-Brown, 2001). A segunda dimensão é centrada na satisfação das necessidades dos indivíduos por parte da organização (needs-supplies fit), um dos aspectos complementares do ajuste (Kristof, 1996). A terceira dimensão, por fim, é relacionada ao atendimento das demandas organizacionais por parte do indivíduo apoiado na existência das habilidades necessárias (demands-abilities fit), o segundo aspecto complementar do ajuste (Kristof, 1996; Cable \& Judge, 1996).

No estudo de Cable e DeRue (2002), as percepções de AIO enquanto congruência de valores (ajuste por similaridade) foram relacionadas a resultados organizacionais, tais como identificação organizacional, comportamento de cidadania organizacional e decisões de rotatividade, enquanto que as percepções de adequação entre necessidades e suprimentos foram relacionadas a atitudes no trabalho, tais como satisfação e comprometimento. A adequação entre habilidades e demandas não foi relacionada aos efeitos hipotetizados no estudo.

Em relação à conexão entre motivação individual e comportamentos considerados fundamentais aos desempenhos individual e organizacional, alguns estudos têm associado MSP ao Comportamento de Cidadania Organizacional (CCO). De acordo com o estudo meta-analítico de Harari et al. (2017), os indivíduos com foco no coletivo possuem objetivos compartilhados e estão inclinados à cooperação e à manifestação de comportamentos discricionários na organização. Nesse entendimento, em estudo realizado na Coreia do Sul, Kim (2006) indicou que os funcionários públicos com maior nível de MSP são comprometidos com o bem público, seguem condutas pautadas pela benevolência, buscam servir aos demais cidadãos e possuem o desejo de influenciar sua comunidade. Além disso, atuam no alcance de objetivos políticos para melhorar o bem público e, voluntariamente, assumem responsabilidades extras e se envolvem em comportamentos pró-sociais.

Organ (1988) definiu o CCO como um comportamento individual discricionário, não reconhecido diretamente ou explicitamente pelo sistema de recompensa formal, que promove o efetivo funcionamento da organização. A formulação desse conceito assentou-se nos estudos de Barnard (1938), sobretudo em relação ao conceito de "vontade de cooperar", e Katz e Kahn (1978), em relação à diferenciação entre o desempenho da função habitual e os comportamentos considerados inovadores e espontâneos (Podsakoff, MacKenzie, Paine, \& Bachrach, 2000).

Mais tarde, Organ (1997) buscou uma reconstrução para o conceito de CCO. Por "discricionário" o autor quis dizer que esse comportamento não é um requisito obrigatório exigido do trabalho ou da descrição do cargo, mas uma escolha pessoal. Recompensas decorrentes de CCO seriam, no máximo, aleatórias e indiretas em comparação com as recompensas decorrentes de contribuições formais. Quanto à efetividade, Organ (1997) aponta que nem todo CCO faz a diferença nos resultados organizacionais. Por tais motivos, o autor passa a conceber o CCO como sinônimo de desempenho contextual associado a contribuições para a manutenção e aprimoramento do contexto social e psicológico que dá suporte ao desempenho das tarefas na organização.

Os estudos sobre CCO distinguem várias dimensões para a compreensão do fenômeno. De acordo com Organ (1988), o CCO abarca altruísmo (por exemplo, ajudar outra pessoa em tarefa ou problema organizacional relevante), cortesia (por exemplo, despender esforços para prevenir um problema ou tomar medidas antecipadamente para mitigá-lo), conscienciosidade (por exemplo, apresentar comportamentos relacionados ao seu papel formal que superem os níveis mínimos exigidos), esportivismo (por exemplo, abster- 
se de queixas insignificantes) e virtude cívica (por exemplo, participar de forma responsável, preocupandose com a vida da organização).

Uma segunda grande dimensionalidade de CCO foi proposta por Williams e Anderson (1991). Esses autores classificaram o $\mathrm{CCO}$ em duas categorias, com base no alvo ou direção do comportamento. Mais especificamente, eles definiram o Comportamento de Cidadania Organizacional orientado à Organização (CCO-O) e o Comportamento de Cidadania Organizacional orientado para outros Indivíduos (CCO-I). Todas as dimensões de CCO dispostas por Organ (1988) podem ser capturadas pelo esquema conceitual de Williams e Anderson (1991). Enquanto as dimensões altruísmo e cortesia podem ser associadas ao CCO-I, as dimensões conscienciosidade, virtude cívica e esportivismo podem ser associadas ao CCO-O (Andrade, 2017; Podsakoff et al., 2000; Podsakoff et al., 2014).

Lee e Allen (2002), assim como Williams e Anderson (1991), consideraram a distinção entre CCO$\mathrm{O}$ e CCO-I. Sendo assim, em revisão à teoria e aos modelos existentes, esses autores desenvolveram oito novos itens para cada um dos tipos de CCO. Enquanto o CCO- I envolve itens voltados à cooperação entre colegas de trabalho, o CCO-O refere-se a comportamentos de melhoria da imagem, defesa, lealdade e desenvolvimento da organização. A revisão de itens realizada por Lee e Allen (2002) teve por intuito abranger comportamentos que são claramente benéficos aos indivíduos e à organização, sendo evitada a sobreposição com o comportamento desviante no trabalho, situação sinalizada para o modelo de Williams e Anderson (1991).

Compreende-se, portanto, que o CCO pode desencadear resultados positivos nas organizações. No setor público não é diferente, na medida em que esses comportamentos podem contribuir para a melhoria dos serviços prestados, superando os males da burocracia e incentivando o desempenho nas diversas unidades de trabalho (Vigoda-Gadot \& Beeri, 2011).

No entanto, ainda são inscipientes os estudos sobre CCO no setor público, fato considerado paradoxal por Vigoda-Gadot e Beeri (2011), uma vez que a expressão cidadania é uma terminologia básica na ciência política, podendo ser somada à Nova Administração Pública por esta enfatizar o papel das pessoas na construção de uma governança efetiva. $\mathrm{O} C \mathrm{CO}$, então, relaciona-se com os objetivos e a visão da administração pública moderna, despertando para investigações sobre seus antecedentes nas organizações.

$\mathrm{Na}$ atualidade, Organ (2018) sugere a realização de estudos empíricos sobre CCO em locais de trabalho não norte-americanos, a fim de que possam ser identificadas diferenças na sua dimensionalidade e nos seus impactos na eficácia organizacional. Além disso, o estudo de realidades distintas pode permitir a identificação de novos correlatos e antecendentes desse comportamento.

\section{PROPOSIÇÃO DO FRAMEWORK DE ANÁLISE DE CONSEQUENTES DE MSP}

Primeiramente, destaca-se que a realização de consultas à produção científica internacional relativa à Motivação do Serviço Público (MSP) foi fundamental para o estabelecimento do framework de análise proposto. Por meio dessas consultas foi possível a definição de potenciais construtos consequentes que podem ser profícuos ao contexto das organizações públicas brasileiras: O Ajuste Indivíduo-Organização (AIO) e o Comportamento de Cidadania Organizacional (CCO).

Ao examinar as origens teóricas, os conceitos, os modelos desenvolvidos, as dimensionalidades dos construtos e os antecedentes e consequentes identificados em estudos anteriores, foi possível o estabelecimento de um modelo de análise para investigações empíricas no contexto brasileiro, o qual é apresentado na Figura 01. 


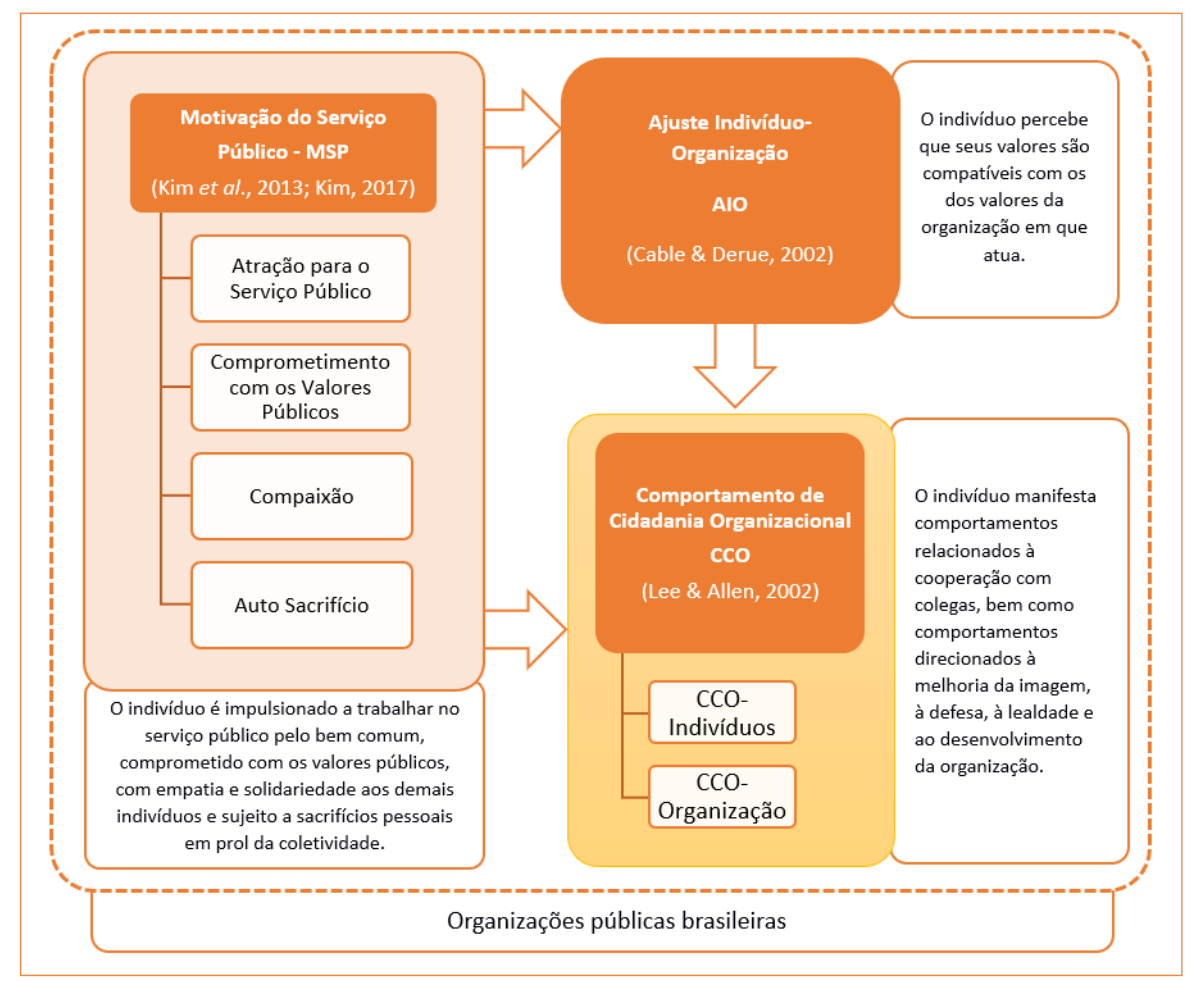

Figura 01. Framework de análise proposto

Fonte: elaborada pelos autores

Em decorrência da formulação do framework ilustrado pela Figura 01, proposições são apresentadas na sequência com a finalidade de subsidiar processos futuros de validação empírica.

Relativamente à abordagem da MSP no Brasil, Buiatti (2007) realizou o processo de validação da escala de mensuração formulada por Perry (1996), porém, seus resultados indicaram a necessidade de revisar a operacionalização dos itens, bem como a contextualização das dimensões de MSP no país. Sendo assim, propóe-se que a análise da MSP no contexto brasileiro ocorra a partir dos pressupostos oferecidos pelos estudos de Kim e Vandenabeele (2010), Kim et al. (2013) e Kim (2017).

Em superação ao modelo de Perry (1996), Kim e Vandenabeele (2010) assinalaram a necessidade de reconstrução do instrumento de mensuração de MSP, a fim de que o mesmo possa ser validado internacionalmente com menos modificações. Esses autores inferem que a MSP é permeada pela noção de auto sacrifício e está associada a três tipos aos motivos: Instrumental, baseado em valores e de identificação.

A partir dessas constatações, Kim et al. (2013) desenvolveram um modelo de mensuração pelo qual a MSP foi composta por quatro dimensões: Atração para o Serviço Público (ASP), Comprometimento com os Valores Públicos (CVP), Compaixão (COM) e Auto sacrifício (AS), cuja composição de itens foi confirmada em 12 países. Posteriormente, Kim (2017) buscou atingir melhores propriedades psicométricas para essa dimensionalidade em pesquisa realizada no contexto coreano. Em decorrência, alguns itens da escala concebida por Kim et al. (2013) foram revisados para melhor adaptação ao contexto institucional do país, aprimorando as propriedades psicométricas do instrumento em diferentes amostras utilizadas na pesquisa.

Em relação ao Ajuste Indivíduo-Organização (AIO), opta-se por considerar a dimensão referente à congruência entre valores individuais e valores organizacionais, oferecida por Cable e DeRue (2002). Seus itens mensuram o ajuste percebido de compatibilidade por similaridade e associam-se principalmente a resultados organizacionais, tais como identificação organizacional, comportamentos de cidadania organizacional e decisões de rotatividade. 
De acordo com Perry e Wise (1990), quanto maior a MSP de um indivíduo, maior a atração por organizações públicas que compreendam suas motivações de serviço público. Uma vez que as organizações do setor público são encarregadas de promover o bem-estar geral da sociedade, presume-se que sua força de trabalho incluirá indivíduos com valores semelhantes, buscando oportunidades para satisfazer seus motivos altruístas em servir ao público (Wright, 2007).

Sendo assim, pode-se argumentar que os indivíduos com altos níveis de MSP são mais propensos do que outros a ter valores e objetivos semelhantes aos da organização que oferece serviços públicos (Jin, Mcdonald, \& Park, 2018). Conforme Christensen e Wright (2011), a MSP promove a congruência entre os valores dos servidores públicos e as missões das organizações públicas.

Portanto, a MSP pode ser uma estratégia eficaz para recrutar pessoas que estarão naturalmente orientadas para os ambientes de trabalho do serviço público, pois, de acordo com estudos de Bright (2008; 2013), os funcionários públicos com altos níveis de MSP foram significativamente compatíveis com as características de sua organização.

Esses apontamentos são também consistentes com os estudos empíricos de Gould-Williams et al. (2015) e Jin et al. (2018), os quais identificaram uma influência direta (positiva e significativa) da MSP sobre o AIO em pesquisas realizadas junto a funcionários públicos egípcios e professores do ensino superior norte-americanos, respectivamente. Esse contexto interacional permitiu o desenvolvimento da primeira proposição:

P1: A Motivação do Serviço Público (MSP) influencia positivamente o Ajuste Indivíduo-Organização (AIO) no setor público brasileiro.

Em relação ao Comportamento de Cidadania Organizacional (CCO), considera-se o modelo de Lee e Allen (2002), o qual é uma revisão do modelo de Willians e Anderson (1991) que avalia duas dimensões ou perspectivas: CCO orientado à organização (CCO-O) e CCO orientado aos indivíduos (CCO-I).

Ainda que a MSP possa ser vista como um valor ou atitude que motiva os indivíduos a se envolverem em comportamentos que beneficiam a sociedade, entende-se que os comportamentos dos funcionários orientados para o serviço público também beneficiarão a organização e seus colegas de trabalho (GouldWilliams et al., 2015).

Assim, uma vez que os funcionários com altos níveis de MSP estão dispostos a trabalhar no serviço público de forma significativa pelo bem comum, comprometidos com os valores públicos, com empatia e solidariedade aos demais indivíduos e dispostos a sacrifícios pessoais em prol da sociedade (Kim et al., 2013), é possível que manifestem o CCO com mais frequência, tanto em relação à organização, quanto aos demais indivíduos (Kim, 2006), despendendo esforços que superem suas atividades rotineiras e formais (Koumenta, 2015).

Estudos internacionais recentes evidenciaram a influência da MSP sobre o CCO em diferentes contextos (Kim, 2006; Cun, 2012; Ferdousipour, 2016; Gould-Williams et al., 2015; Koumenta, 2015; Shim \& Faerman, 2017; Jin et al., 2018), constatação que deu origem à seguinte proposição para o contexto brasileiro:

P2: A Motivação do Serviço Público (MSP) influencia positivamente o Comportamento de Cidadania Organizacional (CCO).

Na sequência, o framework proposto busca elucidar a influência do AIO sobre o CCO, bem como o efeito indireto que o primeiro pode apresentar na interação entre a MSP e o CCO.

Chatman (1989), ao embasar a teoria sobre o AIO, apontou que essa perspectiva de compatibilidade faz com que os indivíduos contribuam de maneira construtiva com seus colegas de trabalho e com a organização.

Wei (2012), a partir da teoria da auto-identidade, infere que o AIO pode moldar o comportamento individual em uma organização. Sendo assim, considera-se que os indivíduos necessitam de um senso de pertencimento e que, quando os mesmos percebem que a cultura e os valores organizacionais estão alinhados com seus valores pessoais e objetivos de carreira, reaçóes emocionais podem levar a comportamentos que extrapolam suas obrigações formais (Jin et al., 2018). 
Portanto, quando os funcionários se identificam com os valores de sua organização, ou seja, quando há um AIO, provavelmente irão contribuir com a missão e os objetivos organizacionais. Nesse sentido, comportamentos para a manutenção e aprimoramento do contexto social e psicológico (CCO) poderão ser direcionados à organização e/ou aos colegas de trabalho com maior frequência e intensidade (Organ, 1997; Cable \& Derue, 2002; Gould-Williams et al., 2015). Consequentemente, foi definida a proposição:

P3: O Ajuste Indivíduo-Organização (AIO) influencia positivamente o Comportamento de Cidadania Organizacional (CCO).

Por fim, compreende-se que a interação entre a MSP e o CCO, além de direta, pode ser influenciada por outros elementos. Nesse sentido, conforme sinalizam estudos internacionais, destaca-se o papel do AIO enquanto consequente de MSP e antecedente de CCO (Jin et al., 2018). O estudo de Gould-Williams et al. (2015), por exemplo, identificou que os efeitos positivos da MSP sobre o CCO em funcionários públicos egípcios foram, além de diretos, influenciados pelo grau de congruência entre os valores dos trabalhadores e os da organização. Pressupõe-se que, conforme os funcionários com altos níveis de MSP sentem que os seus valores e objetivos coincidem com os de sua organização (quando há AIO), eles são mais propensos a manifestar o CCO. Tal constatação deu origem à seguinte proposição:

P4: A Motivação do Serviço Público (MSP) possui um efeito indireto e positivo sobre o Comportamento de Cidadania Organizacional (CCO) por meio do Ajuste Indivíduo-Organização (AIO).

Assim sendo, esboça-se a tese de que a MSP influencia positivamente o AIO percebido, de que a MSP influencia positivamente o $\mathrm{CCO}$, de que o AIO influencia positivamente o $\mathrm{CCO}$ e de que a influência positiva da MSP sobre o CCO, além de direta, é mediada pela percepção de AIO. A Figura 02 esquematiza as proposições passíveis de verificação empírica em decorrência do framework de análise proposto.

Figura 02. Proposições teóricas

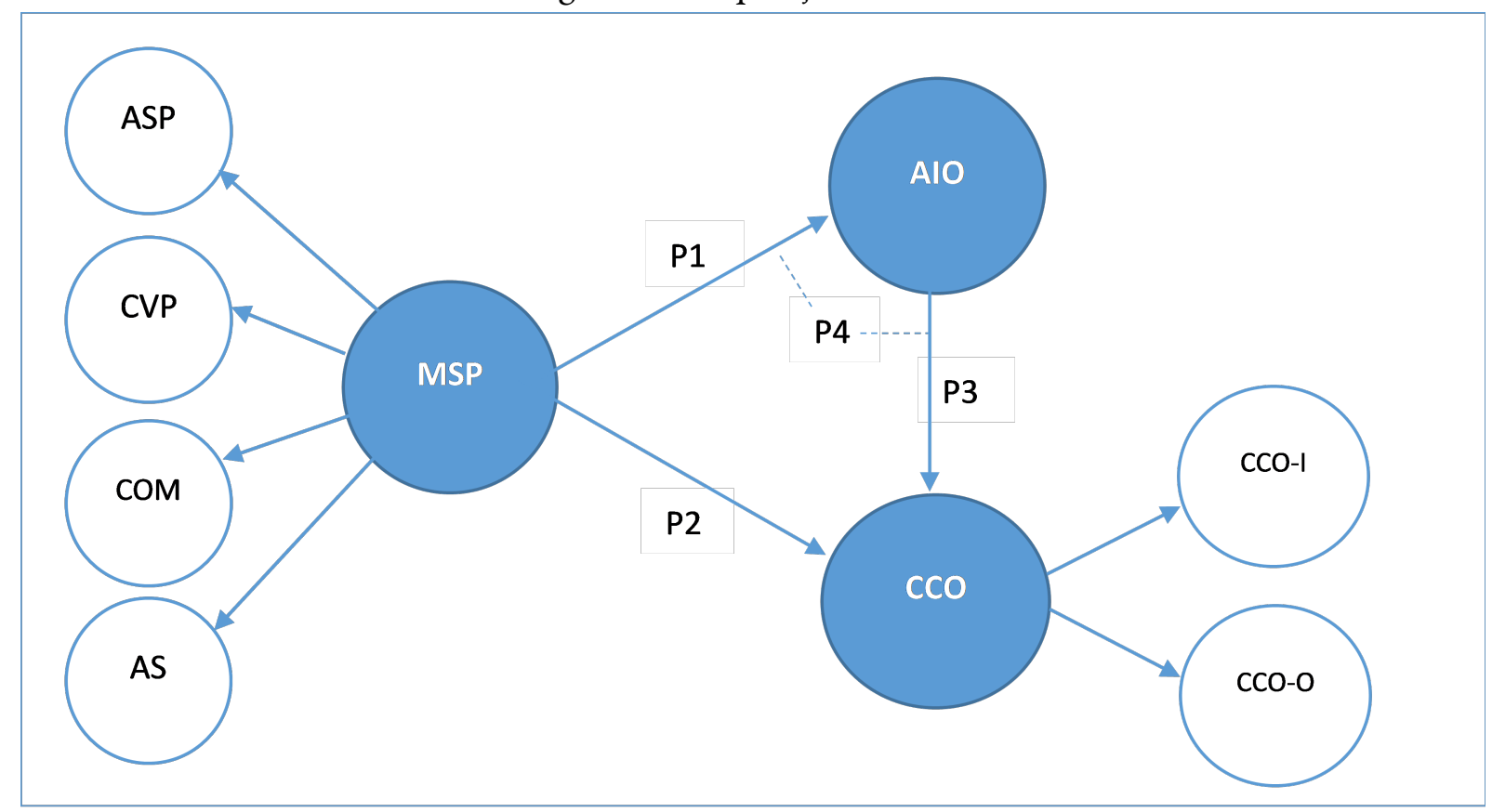

Fonte: elaborada pelos autores

Para que o framework proposto seja efetivado, previamente, sugere-se a utilização de um protocolo de tradução e adaptação de escalas de mensuração (Beaton, Bombardier, Guillemin, \& Ferraz, 2000). Posteriormente, sob a ótica quantitativa, torna-se necessário o emprego de técnicas estatísticas para o processo de validação dos instrumentos de coleta de dados no contexto brasileiro, bem como para a análise de 
influências entres os construtos. Enquanto a Análise Fatorial Confirmatória é indicada para verificar o quão bem a especificação de fatores combina com a realidade, a Modelagem de Equações Estruturais é indicada para explicar as relações entre múltiplas variáveis envolvidas na análise (Hair et al., 2009).

\section{CONSIDERAÇÕES FINAIS}

Este artigo buscou prospectar uma agenda de pesquisa em administração pública, mais especificamente relacionada ao comportamento organizacional em organizações públicas brasileiras. Foram propostos caminhos para a investigação dos reflexos da motivação do serviço público sobre percepções e comportamentos que podem ser benéficos ao ambiente organizacional: $\mathrm{O}$ ajuste indivíduo-organização e o comportamento de cidadania organizacional.

Acredita-se que o framework proposto, quando validado empiricamente, oferecerá subsídios à interpretação do comportamento dos trabalhadores das organizações públicas. Com as proposições oferecidas, torna-se possível avaliar se os indivíduos dotados de motivações pró-sociais associadas ao serviço público (relacionadas ao bem comum, aos valores públicos, à empatia e à assunção de sacrifícios em prol da sociedade) estão ajustados à organização em que atuam (relativamente à compatibilidade de valores) e propensos à manifestação de comportamentos discricionários positivos ao ambiente organizacional (direcionados aos colegas de trabalho e à organização de forma geral).

Por limitação, o presente estudo possui a exposição de proposições oferecidas a partir da teoria, sem o teste empírico em um contexto específico. Por tal motivo, sugere-se que pesquisas futuras sejam realizadas no sentido de validar empiricamente as proposições apresentadas em organizações públicas brasileiras de múltiplas esferas e finalidades, sob diferentes abordagens epsitemológicas e metodológicas. Além disso, aponta-se a possibilidade de desdobramentos do framework proposto de forma a permitir, por exemplo, análises do poder preditivo de cada dimensão de MSP sobre os construtos considerados consequentes.

\section{REFERÊNCIAS}

Andrade, T. (2017). Antecedentes contextuais dos comportamentos de cidadania organizacional (Tese de Doutorado). Universidade Federal de Santa Maria, Santa Maria, RS, Brasil.

Bangcheng, L. (2009). Evidence of public service motivation of social workers in China. International Review of Administrative Sciences, 75(2), 349-366.

Barnard, C. I. (1938). The function of the executive. Cambridge: Harvard University Press.

Beaton, D. E., Bombardier, C., Guillemin, F., \& Ferraz, M. B. (2000). Guidelines for the process of cross-cultural adaptation of self-report measures. Spine, 25(24), 3186-3191.

Becton, J. B., Carr, J. C., Mossholder, K. W., \& Walker, H. J. (2017). Differential effects of task performance, organizational citizenship behavior, and job complexity on voluntary turnover. Journal of Business and Psychology, 32(4), 495-508.

Bellé, N. (2013). Leading to make a difference: A field experiment on the performance effects of transformational leadership, perceived social impact, and public service motivation. Journal of Public Administration Research and Theory, 24(1), 109-136.

Bergue, S. T. (2010). Gestão de pessoas em organizações públicas. Caxias do Sul, RS: Educs.

Brewer, G. A., \& Selden, S. C. (1998). Whistle blowers in the federal civil service: New evidence of the public service ethic. Journal of public administration research and theory, 8(3), 413-440.

Bright, L. (2008). Does public service motivation really make a difference on the job satisfaction and turnover intentions of public employees?. The American Review of Public Administration, 38(2), 149-166. 
Bright, L. (2013). Where does public service motivation count the most in government work environments? A preliminary empirical investigation and hypotheses. Public Personnel Management, 42(1), 5-26.

Buchanan, B. (1975). Red-tape and the service ethic: Some unexpected differences between public and private managers. Administration \& Society, 6(4), 423-444.

Buiatti, C. L. (2007). Validação da escala de PSM - Public Service Motivation por meio de modelagem de equações estruturais (Dissertação de Mestrado). Universidade de São Paulo, São Paulo, SP, Brasil.

Cable, D. M., \& DeRue, D. S. (2002). The convergent and discriminant validity of subjective fit perceptions. Journal of applied psychology, 87(5), 875-884.

Cable, D. M., \& Judge, T. A. (1996). Person-organization fit, job choice decisions, and organizational entry. Organizational behavior and human decision processes, 67(3), 294-311.

Caillier, J. G. (2014). Toward a better understanding of the relationship between transformational leadership, public service motivation, mission valence, and employee performance: A preliminary study. Public Personnel Management, 43(2), 218-239.

Caillier, J. G. (2015). Towards a better understanding of public service motivation and mission valence in public agencies. Public Management Review, 17(9), 1217-1236.

Caillier, J. G. (2015b). Transformational leadership and whistle-blowing attitudes: Is this relationship mediated by organizational commitment and public service motivation?. The American Review of Public Administration, 45(4), 458-475.

Caillier, J. G. (2016). Does public service motivation mediate the relationship between goal clarity and both organizational commitment and extra-role behaviours?. Public Management Review, 18(2), 300-318.

Camilleri, E. (2007). Antecedents affecting public service motivation. Personnel review, 36(3), 356-377.

Carpenter, J., Doverspike, D., \& Miguel, R. F. (2012). Public service motivation as a predictor of attraction to the public sector. Journal of Vocational Behavior, 80(2), 509-523.

Chatman, J. A. (1989). Improving interactional organizational research: A model of person-organization fit. Academy of management Review, 14(3), 333-349.

Chen, C. A., \& Hsieh, C. W. (2015). Knowledge sharing motivation in the public sector: the role of public service motivation. International Review of Administrative Sciences, 81(4), 812-832.

Cheng, K. T. (2015). Public service motivation and job performance in public utilities: An investigation in a Taiwan sample. International Journal of Public Sector Management, 28(4/5), 352-370.

Clerkin, R. M., \& Coggburn, J. D. (2012). The dimensions of public service motivation and sector work preferences. Review of Public Personnel Administration, 32(3), 209-235.

Clerkin, R. M., Paynter, S. R., \& Taylor, J. K. (2009). Public service motivation in undergraduate giving and: Volunteering decisions. The American Review of Public Administration, 39(6), 675-698.

Chatman, J. A. (1989). Improving interactional organizational research: A model of person-organization fit. Academy of management Review, 14(3), 333-349.

Christensen, R. K., \& Wright, B. E. (2011). The effects of public service motivation on job choice decisions: Disentangling the contributions of person-organization fit and person-job fit. Journal of public administration research and theory, 21(4), 723-743.

Crewson, P.E. (1997). Public-service motivation: Building empirical evidence of incidence and effect. Journal of public administration research and theory, 7(4), 499-518.

Cun, X. (2012). Public service motivation and job satisfaction, organizational citizenship behavior: An empirical study based on the sample of employees in Guangzhou public sectors. Chinese Management Studies, 6(2), 330-340.

Edwards, J. R., Cable, D. M., Williamson, I. O., Lambert, L. S., \& Shipp, A. J. (2006). The phenomenology of fit: linking the person and environment to the subjective experience of person-environment fit. Journal of Applied Psychology, 91(4), 802.

Esteve, M., Urbig, D., Van Witteloostuijn, A., \& Boyne, G. (2016). Prosocial behavior and public service motivation. Public Administration Review, 76(1), 177-187. 
Ferdosipour, L. (2016). The relationship among public service motivation, Civic-organizational behavior (obc), and service quality. Iranian Journal of Management Studies, 9(1), 1-20.

Georgellis, Y., \& Tabvuma, V. (2010). Does public service motivation adapt?. Kyklos, 63(2), 176-191.

Gould-Williams, J. S., Mostafa, A. M. S., \& Bottomley, P. (2015). Public service motivation and employee outcomes in the Egyptian public sector: Testing the mediating effect of person-organization fit. Journal of Public Administration Research and Theory, 25(2), 597-622.

Harari, M. B., Herst, D. E., Parola, H. R., \& Carmona, B. P. (2017). Organizational correlates of public service motivation: A meta-analysis of two decades of empirical research. Journal of Public Administration Research and Theory, 27(1), 68-84.

Hair, J. F., Black, W. C., Babin, B. J., Anderson, R. E., \& Tatham, R. L. (2009). Análise multivariada de dados. 6. ed. Porto Alegre: Bookman Editora.

Houston, D. J. (2005). "Walking the walk" of public service motivation: Public employees and charitable gifts of time, blood, and money. Journal of Public Administration Research and Theory, 16(1), 67-86.

Jin, M. H., McDonald, B., \& Park, J. (2018). Does public service motivation matter in public higher education? Testing the theories of person-organization fit and organizational commitment through a serial multiple mediation model. The American Review of Public Administration, 48(1), 82-97.

Katz, D., Kahn, R. L. (1978). Psicologia social das organizações. 2 ed. São Paulo: Atlas.

Kim, S. (2006). Public service motivation and organizational citizenship behavior in Korea. International journal of manpower, 27(8), 722-740.

Kim, S. (2011). Testing a revised measure of public service motivation: Reflective versus formative specification. Journal of Public Administration Research and Theory, 21(3), 521-546.

Kim, S. (2012). Does person\#organization fit matter in the public\#sector? Testing the mediating effect of person\# organization fit in the relationship between public service motivation and work attitudes. Public Administration Review, 72(6), 830-840.

Kim, S., \& Vandenabeele, W. (2010). A strategy for building public service motivation research internationally. Public administration review, 70(5), 701-709.

Kim, S., Vandenabeele, W., Wright, B. E., Andersen, L. B., Cerase, F. P., Christensen, R. K., ... \& Palidauskaite, J. (2013). Investigating the structure and meaning of public service motivation across populations: Developing an international instrument and addressing issues of measurement invariance. Journal of Public Administration Research and Theory, 23(1), 79-102.

Kim, S. (2017). Developing an item pool and testing measurement invariance for measuring public service motivation in Korea. International Review of Public Administration, 22(3), 231-244.

Knoke, D., Wright-Isak, C. (1982). Individual motives and organizational incentive systems. Research in the Sociology of Organizations, 1(2), 209-254.

Koumenta, M. (2015). Public service motivation and organizational citizenship. Public Money \& Management, 35(5), 341-348.

Kristof, A. L. (1996). Person\#organization fit: An integrative review of its conceptualizations, measurement, and implications. Personnel psychology, 49(1), 1-49.

Lauver, K. J., \& Kristof-Brown, A. (2001). Distinguishing between employees' perceptions of person-job and personorganization fit. Journal of vocational behavior, 59(3), 454-470.

Lee, K., \& Allen, N. J. (2002). Organizational citizenship behavior and workplace deviance: The role of affect and cognitions. Journal of applied psychology, 87(1), 131.

Liu, B., Tang, T. L. P., \& Yang, K. (2015). When does public service motivation fuel the job satisfaction fire? The joint moderation of person-organization fit and needs-supplies fit. Public Management Review, 17(6), 876-900.

Liu, B., \& Perry, J. L. (2016). The psychological mechanisms of public service motivation: A two-wave examination. Review of Public Personnel Administration, 36(1), 4-30. 
Liu, B., Zhang, X., Du, L., \& Hu, Q. (2015). Validating the construct of public service motivation in for-profit organizations: A preliminary study. Public Management Review, 17(2), 262-287.

Naff, K. C., \& Crum, J. (1999). Working for America: Does public service motivation make a difference?. Review of public personnel administration, 19(4), 5-16.

Organ, D. W. (1988). Organizational citizenship behavior: The good soldier syndrome. Lexington: Lexington Books.

Organ, D. W. (1997). Organizational citizenship behavior: It's construct clean-up time. Human performance, 10(2), 85-97.

Organ, D. W. (2018). Organizational citizenship behavior: Recent trends and developments. Annual Review of Organizational Psychology and Organizational Behavior, 80, 295-306.

Pandey, S. K., Wright, B. E., \& Moynihan, D. P. (2008). Public service motivation and interpersonal citizenship behavior in public organizations: Testing a preliminary model. International Public Management Journal, 11(1), 89-108.

Park, S. M., \& Rainey, H. G. (2008). Leadership and public service motivation in US federal agencies. International public management journal, 11(1), 109-142.

Pedersen, M.J. (2013). Public service motivation and attraction to public versus private sector employment: Academic field of study as moderator?. International Public Management Journal, 16(3), 357-385.

Perry, J. L., \& Wise, L. R. (1990). The motivational bases of public service. Public administration review, 367-373.

Perry, J. L. (1996). Measuring public service motivation: An assessment of construct reliability and validity. Journal of public administration research and theory, 6(1), 5-22.

Piatak, J. S. (2016). Public service motivation, prosocial behaviours, and career ambitions. International Journal of Manpower, 37(5), 804-821.

Podsakoff, P. M., MacKenzie, S. B., Paine, J. B., \& Bachrach, D. G. (2000). Organizational citizenship behaviors: A critical review of the theoretical and empirical literature and suggestions for future research. Journal of management, 26(3), 513-563.

Podsakoff, N. P., Podsakoff, P. M., MacKenzie, S. B., Maynes, T. D., \& Spoelma, T. M. (2014). Consequences of unit\#level organizational citizenship behaviors: A review and recommendations for future research. Journal of Organizational Behavior, 35(S1), S87-S119.

Rainey, H. G. (1982). Reward preferences among public and private managers: In search of the service ethic. The American Review of Public Administration, 16(4), 288-302.

Riba, C., \& Ballart, X. (2016). Public Service Motivation of Spanish High Civil Servants. Measurement and Effects. Revista Española de Investigaciones Sociológicas (REIS), 154(1), 65-99.

Ritz, A. (2009). Public service motivation and organizational performance in Swiss federal government. International review of administrative sciences, 75(1), 53-78.

Saks, A. M., \& Ashforth, B. E. (1997). A longitudinal investigation of the relationships between job information sources, applicant perceptions of fit, and work outcomes. Personnel psychology, 50(2), 395-426.

Shim, D. C., \& Faerman, S. (2017). Government employees' organizational citizenship behavior: The impacts of public service motivation, organizational identification, and subjective OCB norms. International Public Management Journal, 20(4), 531-559.

Scott, P. G., \& Pandey, S. K. (2005). Red tape and public service motivation: Findings from a national survey of managers in state health and human services agencies. Review of Public Personnel Administration, 25(2), 155-180.

Simone, S., Cicotto, G., Pinna, R., \& Giustiniano, L. (2016). Engaging public servants: Public service motivation, work engagement and work-related stress. Management Decision, 54(7), 1569-1594.

Steijn, B. (2008). Person-environment fit and public service motivation. International public management journal, $11(1), 13-27$.

Schwarz, G., Newman, A., Cooper, B., \& Eva, N. (2016). Servant leadership and follower job performance: The mediating effect of public service motivation. Public Administration, 94(4), 1025-1041. 
Taylor, J. (2008). Organizational influences, public service motivation and work outcomes: An Australian study. International Public Management Journal, 11(1), 67-88.

Taylor, J. (2014). Public service motivation, relational job design, and job satisfaction in local government. Public Administration, 92(4), 902-918.

Teo, S. T., Pick, D., Xerri, M., \& Newton, C. (2016). Person-organization fit and public service motivation in the context of change. Public Management Review, 18(5), 740-762.

Thompson, J. A., \& Christensen, R. K. (2018). Bridging the public service motivation and calling literatures. Public Administration Review, 78(3), 444-456.

Tuan, L. T. (2016). How servant leadership nurtures knowledge sharing: The mediating role of public service motivation. International Journal of Public Sector Management, 29(1), 91-108.

Van Loon, N. M. (2016). Is public service motivation related to overall and dimensional work-unit performance as indicated by supervisors?. International Public Management Journal, 19(1), 78-110.

Vandenabeele, W. (2007). Toward a public administration theory of public service motivation: An institutional approach. Public management review, 9(4), 545-556.

Vandenabeele, W. (2008). Government calling: Public service motivation as an element in selecting government as an employer of choice. Public administration, 86(4), 1089-1105.

Vigoda-Gadot, E., \& Beeri, I. (2011). Change-oriented organizational citizenship behavior in public administration: The power of leadership and the cost of organizational politics. Journal of Public Administration Research and Theory, 22(3), 573-596.

Wei, Y. C. (2012). Person-organization fit and organizational citizenship behavior: Time perspective. Journal of Management \& Organization, 18(6), 833-844.

Williams, L. J., \& Anderson, S. E. (1991). Job satisfaction and organizational commitment as predictors of organizational citizenship and in-role behaviors. Journal of management, 17(3), 601-617.

Wright, B. E. (2007). Public service and motivation: Does mission matter?. Public administration review, 67(1), 54-64.

Wright, B. E., Christensen, R. K., \& Isett, K. R. (2013). Motivated to adapt? The role of public service motivation as employees face organizational change. Public Administration Review, 73(5), 738-747.

Wright, B. E., Hassan, S., \& Park, J. (2016). Does a public service ethic encourage ethical behaviour? Public service motivation, ethical leadership and the willingness to report ethical problems. Public Administration, 94(3), 647-663.

Wright, B. E., \& Pandey, S. K. (2008). Public service motivation and the assumption of person-Organization fit: Testing the mediating effect of value congruence. Administration \& Society, 40(5), 502-521.

Wright, B. E., Moynihan, D. P., \& Pandey, S. K. (2012). Pulling the levers: Transformational leadership, public service motivation, and mission valence. Public Administration Review, 72(2), 206-215.

\section{BY-NC-ND}

